\title{
Cross-linguistic experimental evidence distinguishing the role of context in disputes over taste and possibility
}

\author{
E. Allyn Smith, Elena Castroviejo, and Laia Mayol \\ UQÀM (Montréal), EHU/UPV (Vitoria-Gasteiz), and UPF (Barcelona) \\ smith.eallyn@uqam.ca
}

\section{Introduction}

'Faultless disagreement' is exemplified in (1) and (2) for predicates of personal taste (PPT), where the intuition of philosophers and linguists has been that Sam is expressing his opinion about the cake and that Sue, in saying $N_{o}$, is either expressing that the the cake does not taste good to her or expressing that the cake should not be considered tasty more generally (following [3] and [4], i.a.).

Mary: How's the cake? Sam: It's tasty.

Sue: No it isn't, it tastes terrible!

Mary: How's the cake? Sam: It tastes good to me.

Sue: \#No it isn't/doesn't, it tastes terrible!

One might think that Sam's utterance in (1) is a subjective one, essentially expressing that he personally finds the cake tasty, in which case one would not expect significant meaning differences between (1) and a similar utterance where the subjectivity is made explicit, such as (3). However, [5], arguing for a modification of [6], points out that when PPTs are explicitly relativized to the speaker, it is no longer felicitous to use $N o$ to directly dispute utterances in which they appear, as shown in (4). Stephenson draws a parallel between PPTs and epistemic modals (EMs) such as might, as in (5)-(8), on the basis of their behavior when followed by $N o$, where the addition of $I$ don't know to the utterance makes the uncertainty of the modal explicit. ${ }^{1}$ The general idea is that both PPTs and EMs change behavior with speaker-restriction: in PPTs, the restriction tells us whose tastes are represented, and in EMs, it tells us whose knowledge is the basis for the statement (following a Kratzerian analysis of modals). ${ }^{2}$

\footnotetext{
${ }^{1}$ She actually uses I don't know that he isn't, which we simplified for our experiment given the known difficulty of multiple negations for naive speaker judgments as well as difficulty translating into languages where the literal form is ungrammatical.

2 A reviewer points out that the pattern in (7) and (8) is not necessarily replicated with other ways of relativizing to the speaker, such as As far as I know..., an intuition we share but did not test in our experiment. This evidence supports our same general conclusion that PPTs and EMs are perhaps not as parallel as they first appear.
} 
Mary: Where's Bill? Sam: He might be in his office

Sue: No, he can't be. He doesn't work on Fridays.

Mary: Where's Bill? Sam: I dont know whether he's in the office.

Sue: \#No, he isn't/can't be, he doesn't work on Fridays.

Given the importance of these data to subsequent work in the field across a variety of disciplines and phenomena (including the contextualist/relativist debate, the treatment of polarity particles, and the use of refutation as a diagnostic elsewhere, such as for presupposition projection, just to name a few), we tested whether this pattern could be observed behaviorally in the perceptions of English speakers and whether it could be replicated cross-linguistically in French, Spanish, and Catalan (though for reasons of space, we will only discuss the English and Spanish results here). A better understanding of the psycholinguistic patterns of these "disagreements" is additionally important if they are to be used in computational or other applications given that, up to this point, they have been considered as patterns arising from linguistic structure in context and not, for example, as arising from individual or cultural context differences. The results we present here suggest that there is variability across languages but not across dialects spoken in culturally-different areas, suggesting that the patterns in (1)-(8) are indeed a product of linguistic structure and context. In order to account for the cross-linguistic differences, however, it will be important to de-couple the analysis of subjectivity in PPTs and EMs.

\section{Taste and Possibility}

Though authors disagree about the location and the number of sources of subjectivity in sentences with PPTs, linguists have mostly modelled these predicates as introducing judge parameters (e.g.[5] [6] [7]). For the purposes of this paper, the details of how each of these theories is formalized and the differences between contextualist and relativist approaches are less important than the fact that, in each, there is a parallel drawn with EMs (cf. [5], [8]). If PPTs evaluate content/truth with respect to someone's taste, then, analogously, EMs evaluate content/truth with respect to someone's knowledge. In both cases, when that person is overtly specified grammatically (to be the speaker) rather than filled in contextually, disagreement should become infelicitous, consistent with the intuitions represented in (4) and (8) above.

[1] gives an account of PPTs and other kinds of evaluative propositions such as those with aesthetic adjectives, and in so doing, draws on [2]. We present the basics of their discourse model and return to Umbach's own analysis in the discussion section. The model contains:

- A common ground, (CG): publicly shared beliefs by all participants.

- A discourse commitment (DC) set, $D C_{X}$, for each participant $X: X$ 's public beliefs that are not (or not yet) shared by all other participants. 
- The Table: similar in role to the Question Under Discussion (QUD) [11]. A stack of syntactic objects paired with their denotations that represent the discourse topic at any given time.

- A projected set of all potential CGs that could result from future additions to the current CG.

- A set of conventions, operations, and update rules for the kinds of moves permitted. For example:

- The Table should not be empty (there should be a question)

- The goal, however, is to empty it (we should answer questions)

- The canonical way of removing an item from the Table is to reach a state in which the issue is decided ( $p$ or $\neg p$ follows from the CG)

- Asserting $p$ adds $p$ to the speaker's DC and proposes adding it to the CG by 'pushing' it onto the Table (raising the question of 'whether $p$ ', which we will call ?p)

- If participants voice no objection, $p$ moves from the DC to the CG

- If someone does voice an objection, several things can happen, including agreeing to disagree, leaving $p$ and $\neg p$ in their $D C_{X}$ s instead of the CG and popping ? $p$ off the Table

This discourse model can account for why many kinds of content may not be directly refuted using $N o$. [2] hypothesizes that $N o$ can only target propositions on the Table at the top of the stack. This accounts for why, for example, presuppositions and conventional implicatures are difficult to reject directly, because they are already in the CG and/or not the main issue at hand (cf. [9] [12] [13]). Here, we are concerned with other kinds of content that cannot be rejected, namely explicitly relativized PPTs (henceforth, PPTR) and explicitly relativized EMs (henceforth, EMR).

Returning to the initial data, the analysis in this kind of model would be something like the following: PPT sentences are ambiguous between a reading where someone is expressing her opinion only and not pushing for $p$ to be added to the CG (in which case that person is just adding something to her DC without putting it on the Table) and a reading in which she is additionally suggesting that it should be added to the CG for everyone. Some have mentioned the felicity of Poutine is tasty, but I don't like it, suggesting that one may also put something on the Table to be accepted as a general fact while simultaneously adding its negation to her own DC. PPTR, on the other hand, have only the first of these readings, meaning that they remain exclusively discourse commitments of the speaker and are not pushed onto the Table. Because they are not on the Table, they may not be targeted by $N o$.

One further related concept will be useful for our discussion. [12] define a notion of 'At-issueness' in which a proposition $p$ is at-issue relative to a QUD iff $? p$ is relevant to that QUD. In order for a question ? $p$ to be relevant to a QUD, it must have an answer which contextually entails at least a partial answer to the QUD. In other words, if the question is Who is at the library? and there are four people there, naming all of them is a complete answer, but even giving one or two would be relevant. If someone says Daniel is at the library, then ?p is Is 
Daniel at the library? Either answer $(\mathrm{Y} / \mathrm{N})$ would be relevant to the QUD, thus making the proposition at-issue with respect to this QUD. On this analysis, No targets only at-issue meanings, giving largely the same result as with [2].

\section{$3 \quad$ Experimenting with taste and possibility}

In order to confirm the intuitions presented in (1)-(8) for English and other languages, we created an internet-based audio survey in which participants ranked the relative strangeness of various propositions in response to others across a set of constructed two-turn dialogues. While the fillers were all dialogues of agreement, the critical stimuli contained a refutation with $N o$. We used English as our baseline given the preponderance of English examples in the literature, but we also tested languages from another family, including Spanish, which we focus on here. Hypothesized cross-linguistic differences included the fact that whereas English makes a difference between a polarity particle and sentence negation (No v. not), Spanish only has the lexical item No to carry out the two functions.

Based on the previous literature, the predictions were as follows:

- Disagreement (faultless or not) should be possible with PPTs and EMs, so their average ratings should be as high as that of assertions.

- Relativizing a PPT to a judge should limit its refutability, so the average ratings for PPTRs should be lower than for PPTs.

- EMs and PPTs should show the same pattern (so EMRs $<$ EMs).

- These basic patterns should be consistent across languages.

Stimuli The stimuli consisted of 88 two-turn dialogues. 44 of these dialogues were fillers, 20 were critical items and 24 were items for an experiment not reported in this paper. In the first turn, a statement is made that crucially contains a particular meaning type: PPT, PPTR, EM, and EMR or a fifth, Basic Assertion (BA), as a baseline. In the second turn, for the critical stimuli, participants heard a direct refutation. Example stimuli for the five meaning types in English and Spanish are as follows:

- BA: Daniel is at the library. No, he's at the post office. Dani está en la biblioteca. No, está en correos (Iberian)/el correo (Uruguayan).

- PPT: It's cold outside. No, it is rather warm.

Hace frío afuera. No, hace más bien calor.

- PPTR: Laura's talk was boring to me. No, it was inspiring. A mí la charla de Laura me pareció patética. No, fue estimulante.

- EM: Matthew may join us later. No, he is going on a date. Alberto a lo mejor vendrá (Iberian)/Capaz que Alberto va a venir (Uruguayan) con nosotros más tarde. No, tiene une cita.

- EMR: I don't know whether the kids will take a vacation this year. No, they are going to Corsica.

No sé si los ninos tendrán vacaciones este ano. No, se van a Córcega. 
When translating the stimuli into other languages and dialects, we tried to be as faithful as possible to the original items without ending up with sentences that were vastly less natural than they were in the source language. The stimuli were recorded by two female native speakers in each language; one was always speaker A and the other, B. They read their lists of sentences separately and we spliced them together into dialogues afterwards. We chose audio rather than written presentation of stimuli to avoid the possibility that, when reading, participants might mentally assign different intonational contours and interpret $N o$ responses more felicitously via metalinguistic negation. We only included takes with falling intonation at the end of both turns.

Procedure The experiment was administered via an internet survey platform called SurveyGizmo. Participants were asked to listen to short audio files containing one statement followed by either an acceptance or refutation of some part of the meaning of that statement and answer the following question: "How strange do you think it would be for someone to respond this way?" Participants saw the question and were asked to respond using a 7-point Likert scale from 'very strange' to 'not at all strange'. We chose to ask about how strange they thought it might be for someone to respond this way because it allowed us not to train our participants in the notion of entailment or give away the goal of the experiment. We were careful to focus on how strange the response was to cut down on judgments of the oddness of the initial sentence by itself, though we also conducted a second task (described in the Norming subsection below) to guard against this possibility as well. In addition to screens where they played a sound file and responded to the question of interest, after some of the items, participants had to answer a comprehension question. These questions were added to ensure that participants were paying attention, and the data of those who did not answer correctly was not analyzed. After the experiment, participants were asked to answer a feedback question about what they thought the experiment was about. The great majority of the participants did not show any awareness of the goal of the experiment.

Participants The data presented here come from 55 American English speakers, 60 Iberian Spanish speakers, and 70 Uruguayan Spanish speakers. All were native speakers of the language in which they were tested. The places from which they were recruited were Chicago, Madrid, and Montevideo, respectively. The English participants fulfilled course requirements by participating, while the Spanish speakers in both locations, though largely also students, were entered for a chance to win one of several gift cards.

Norming task Since our stimuli were extremely varied, a concern that one might have is that our participants were not rating how good the reply was, but how marked or frequent the first sentence was. This was especially a concern given that some types of stimuli were longer than others. In order to be able to distinguish the markedness of the first sentence from the appropriateness of the reply, we carried out a baseline task in each language with 16 to 32 participants. The 
procedure was the same as explained above, except that participants only heard the first sentence of any given dialogue, so they did not hear the reply. Then they were asked to rate them in a 7-point Likert scale answering the following question: "How strange do you think it would be for someone to say this?" The participants who participated in the baseline task did not participate in the other experiment. These ratings were then incorporated into our statistical model as described in the next section.

\section{Results}

All analyses are best-fit linear mixed effects models and were computed in R [15] using the lmer function in the lme4 package [16]. The variable whose behavior we were trying to predict was the normalized Likert score that participants gave as a measure of felicity. The fixed effects included the rating of the felicity of the item without a refutation from the norming task and, when comparing meaning subtypes to one another, the meaning type. As for the random effects, the best models were always those that took item and subject differences into account (i.e. random intercepts for 'Item' and 'Participant'), and occasionally the best model was one that included the by-subject random slope for meaning subtype (which accounts for any adjustments to the effect of 'Subtype' by a difference between participants). What follows are plots of the normalized means.

\section{English Judgments}

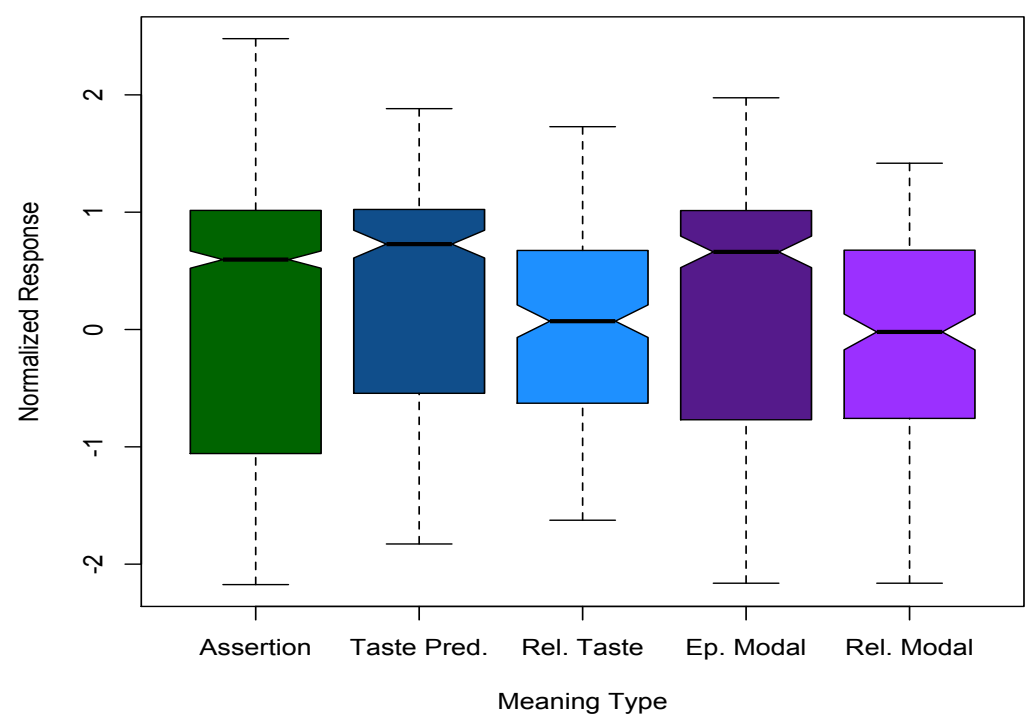


English results The figure above matches the statistical results: there is no significant difference between the means of assertion, PPTs and EMs. Refutation of PPTs was significantly better $(\mathrm{p}<.01)$ than refutation of PPTRs. Refutation of EMs was significantly better $(\mathrm{p}<.01)$ than refutation of EMRs. In other words, the intuitions in the literature are perfectly borne out in the English data.

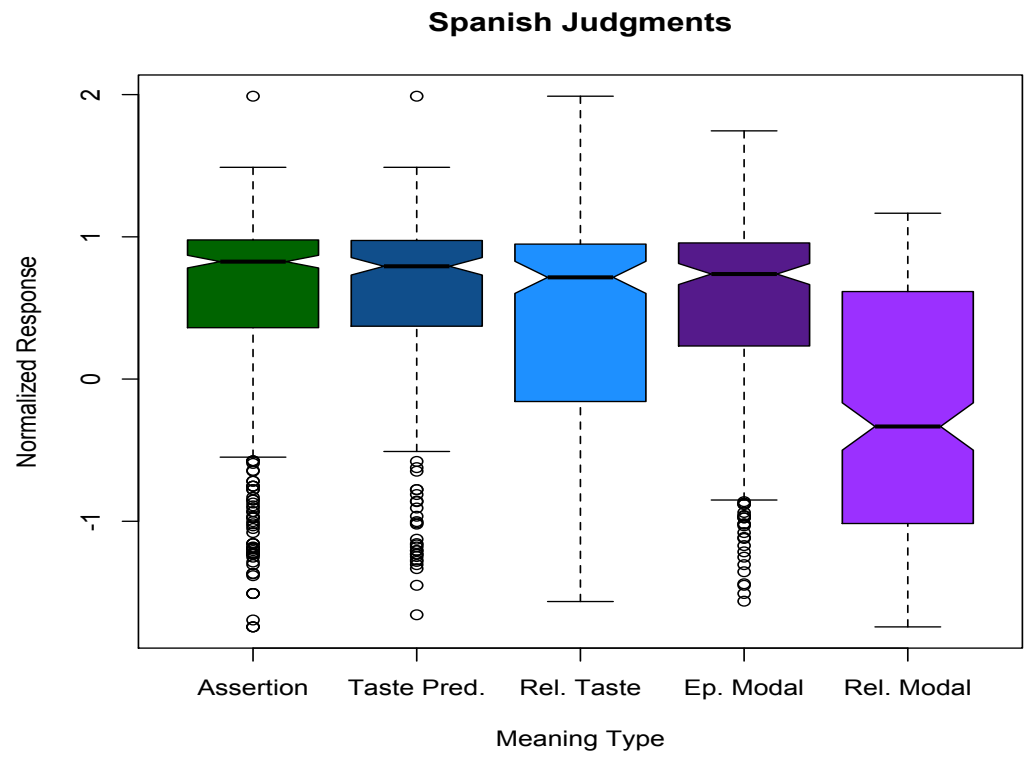

Iberian Spanish results This figure again matches the statistical results: there is no significant difference between the means of assertion, PPTs and EMs. Refutation of PPTs was not significantly different from refutation of PPTRs. Refutation of EMs was significantly better $(\mathrm{p}<.01)$ than refutation of EMRs. In other words, Iberian Spanish speakers can directly refute PPTs and PPTRs as well as EMs, but not EMRs. These results are problematic for theories arguing for a parallel between EMs and PPTs with respect to a judge parameter or implicit argument, and they are surprising if we think that disagreement should be able to tease apart PPTs from PPTRs cross-linguistically.

\subsection{Possibility 1: Cultural Context}

In English, disagreements with BAs, PPTs and EMs are well rated, while disagreements with PPTRs and EMRs are not, which is consistent with the idea that an explicit judge experiencer argument blocks direct refutation. But in Spanish, disagreements with BAs, PPTs, PPTRs and EMs are well rated, while 
refuting an EMR sentence is not. Thus, we need to give an account for why PPTRs can be followed by direct disagreement in Spanish but not English. One possibility is that this is a question not of the grammar/linguistic context per se but of whether people in a given culture feel comfortable arguing with other people's judgments. After all, it is possible to say No, the soup is not tasty to you, you're lying to spare the cook's feelings, so participants could potentially hear the No in our stimuli as being anaphoric to something like that such that they understand it to be No, the soup is not tasty to you, you're lying to spare the cook's feelings; it tastes terrible!. Arguing with someone about his/her own tastes is certainly impolite in English and has been called a 'faulty disagreement' by [17] who consider it infelicitous (though personally, the first author of this paper feels that there is a difference between such a disagreement and ones like (4)). But it is possible that this would not be seen as a violation of politeness norms in Iberian Spanish. If we had independent evidence that speakers of a language (in this case, Spanish) are more indirect in one area as compared to another, then re-running the same experiment with the other population should change the results. Such evidence exists for Iberian v. Uruguayan Spanish, from anthropologists and linguists such as [18]. Uruguayan Spanish is claimed to be much more indirect than Iberian Spanish, especially in terms of requests, but also criticism, which would likely decrease not only the rates with PPTR, but perhaps the felicity of PPTs and other categories as well. As we see in the figure below, however, the Uruguayan Spanish speakers have an identical pattern to the Iberian speakers.

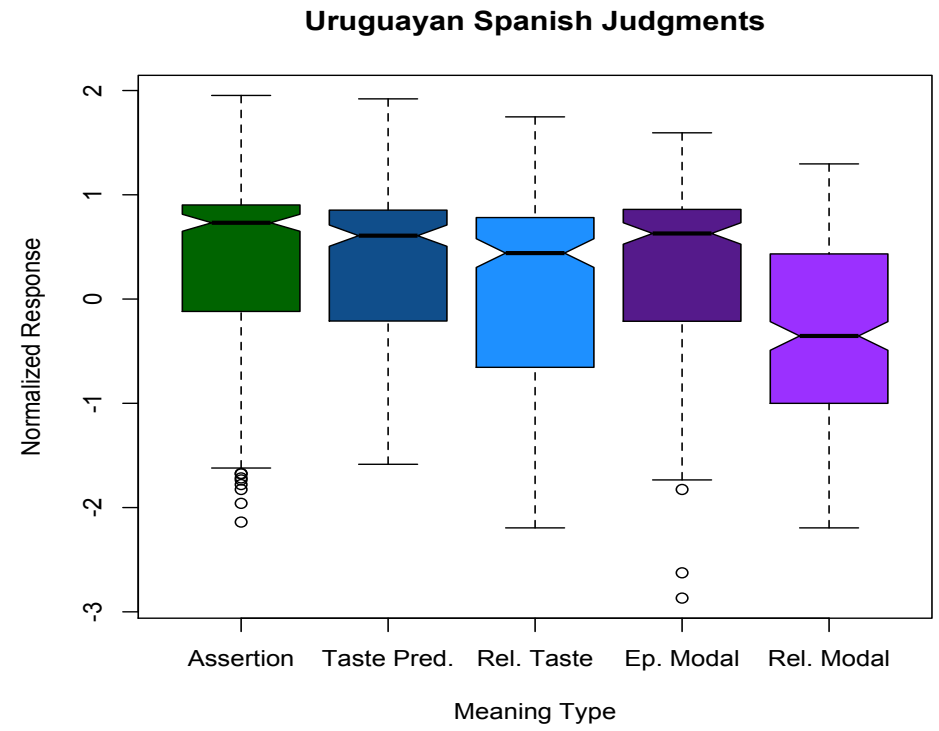


In addition to verifying the same significant differences or lack thereof (between PPTs and PPTRs), we also conducted a series of t-tests comparing each category across the two dialects, resulting in no significant differences. We take this as evidence that the cross-linguistic difference in acceptability of No with PPTR is based on the linguistic context (how the grammar interacts with what has been said and affects what will/can be said). This is what we turn to in the next section.

\subsection{Possibility 2: Linguistic Context}

Recall from section 2 that [1], among others, would explain the difference between PPT and PPTR as being that PPT have a reading on which $p$ is pushed to the Table, whereas PPTR do not and stay in the DC of the speaker. No can only target content on the Table. In order to explain why $N o$ in Spanish could target both PPTs and PPRTs, one would need to either hypothesize that No in Spanish is different from $N o$ in English in that it targets propositions from any part of the discourse model, perhaps, or we would need to say that PPTRs in Spanish but not English put $p$ on the Table. Another possibility would be to claim that in Spanish, but not English, accommodation of a proposition not on the Table could take place. We prefer, however, to rethink the definition of what each type of content contributes to the discourse model in the first place, as discussed below.

Returning to [1], Umbach makes two different kinds of distinctions, one which she considers to be semantic, and the other pragmatic. In an earlier version of the paper, the semantic distinction is between descriptive propositions like Daniel is at the library and evaluative propositions like Poutine is tasty. The general idea is that descriptive propositions are asserted as facts, while evaluative propositions represent opinions. Her diagnostic for the difference has to do with whether a given proposition can be embedded under the verb finden 'find' in German (though for the purposes of our examples, English works just as well). While it is fine to say that one finds the soup tasty, it is not possible to say that one finds Daniel to be at the library (or perhaps more clearly, that one finds Ottawa to be the capital of Canada). ${ }^{3}$ In addition to the descriptive/evaluative distinction, a pragmatic difference is proposed between general and subjective judgments in [1]. General judgments are proposed by the speaker to enter the CG and subjective judgments do not have this requirement (they may enter the CG, but they can also stay in the DC). These two distinctions yield four categories, each corresponding to one of the meaning subtypes we tested (shown below in Table 1). The only one not represented is the EMRs, which we propose are also

\footnotetext{
3 This gets changed in the final version because finden does embed some uses of non-evaluative dimensional adverbs like tall and does not work with some uses of evaluative propositions (the non-meta-linguistic ones, cf.[19]). Because we do not have room to go into the interesting differences among kinds of gradable predicates and are focusing on just one subtype, we ignore this distinction here (see also [20] for further work on whether gradable predicates should be considered PPTs).
} 
descriptive propositions expressing general judgments, as with BAs. We return to this in the justifications below.

\begin{tabular}{|l|l|l|}
\cline { 2 - 3 } \multicolumn{1}{c|}{} & Descriptive propositions & Evaluative propositions \\
\hline General Judg. & BA: Daniel is at the library & PPT: Poutine is tasty \\
\hline Subjective Judg. & EM: Daniel might be at the library & PPTR: Poutine is tasty to me \\
\hline
\end{tabular}

Table 1. Examples instantiating each category of an earlier draft of [1]

In what follows, we use the same distinctions as in [1] but tweak the functions of the categories. We hypothesize that both distinctions are related to discourse moves: the type of proposition determines what is added to the speaker's DC (or, alternatively, the semantics of each of these types leads to such a distinction), and the type of judgment determines what is pushed to the Table. For the purposes of this section, we need to define the following notation: $p$-individual ( $p$-ind) is a proposition relativized to an individual (usually the speaker), and $p$-generic or $p$-general-knowledge ( $p$-gen) is a proposition relativized to society more broadly. In the case of PPT/Rs, the fact that the soup tastes good to the speaker is $p$-ind and the fact that it is considered delicious more generally is $p$-gen.

We propose that descriptive propositions add only a single proposition to the discourse commitment set of the speaker (here we are not counting the join of various propositions as a single proposition, there can be additional propositions contributed by non-restrictive relative clauses, etc., that do not count toward this limit). This proposition is always pushed to the Table and it is the full literal meaning of the sentence. In the case of the BA, that is $p$, and in the case of the EM, that is $\diamond p$ (because $p$ is embedded and so not the full meaning). For EMR, it is the proposition that the speaker has no knowledge of $p$. This is why EMR is categorized with BA: it is a factual statement, it just doesn't assert $p, \neg p$, their disjunction, or anything other than an ignorance claim. Evaluative propositions, on the other hand, by their nature, involve an individualized and generalized version and, thus, add these two propositions to the speaker's DC by default. For PPTs and PPTRs, these are $p$-ind and $p$-gen. The reason that we say they are added 'by default' is because, whereas the descriptive proposition is an assertion, with evaluative propositions, one of the two propositions is an assertion and the other, a conversational implicature (as can be clearly tested using cancelation). Depending on whether the PPT is explicitly relativized or not, which of these ( $p$-ind or $p$-gen) is an implicature changes:

The soup is tasty

...but I don't like it ...\#but I'm the only one who likes it

The soup tastes good to me

...but I'm the only one who likes it ...\#but I don't like it 
It may seem unusual to think of conversational implicatures as default DCs and things that can be put on the Table, but they do seem to be targetable using polarity particles as in A: Is your friend handsome? B: He has a nice personality. $C$ : No, he's good-looking, too, where $C$ responds to $B$ 's implicature. We are additionally proposing that for the implicature in the pair of (evaluative) propositions, it can be either the positive or negative form (depending on what has been cancelled, general knowledge, etc.). For example, in a context where someone says The soup tastes good to me but perhaps not to others, the implicature is that the soup's tastiness will not generally hold up.

One other note about EMRs relates to the notion of at-issueness and relevance to a QUD. It would be easy to conflate the notion of relevance from Grice (and generally the notion that discourses require coherence) with relevance to a QUD, and they are indeed quite similar. However, here we have claimed that $I$ don't know $p$ never introduces $p$ or $\neg p$ to the DC, which means they cannot be pushed onto the Table. It also means that they are not at-issue, because the way the notion of relevance is defined, an answer to a question of whether you have knowledge of $p$ does not eliminate an alternative in the question ? $p$ (of course, it could eliminate an alternative to ?q if that were a question like Do you know whether $p$ ?). However, in the broader sense of relevance, I don't know is clearly a pertinent response to the question. Thus, our analysis is proposing that there are felicitous discourse moves with no at-issue content, simultaneously illustrating one difference between at-issueness and what is on the Table.

The following table summarizes the hypotheses presented above in its first column, and we now turn to the contribution of the different judgment types, shown in the second column.

\begin{tabular}{|l|l|l|}
\cline { 2 - 3 } \multicolumn{1}{c|}{} & Added to the speaker's DC & Pushed to the Table \\
\hline Gen/Desc: BA & $p$ & $p$ \\
\hline Gen/Eval: PPT & $p$-gen and either $p$-ind or $\neg p$-ind & $p$-gen \\
\hline Subj/Desc: EM & $\diamond p$ & $\diamond p$ and $p$ \\
\hline Subj/Eval: PPTR & $p$-ind and either $p$-gen or $\neg p$-gen & $p$-ind and either $p$-gen or $\neg p$-gen \\
\hline
\end{tabular}

Table 2. Update rules for each combination of proposition/judgement type

The essence of our analysis of the difference between general and subjective judgments is that general judgments put just one proposition on the Table, and a general one at that, while subjective judgements push two propositions to the table, giving the more subjective proposition pride of place (the secondary nature of the general proposition ties into Umbach's original distinction). The idea draws on inspiration from [10] who propose that EMs put both $p$ and $\diamond p$ on the Table and that which is targeted as at-issue depends upon various factors (thereby accounting for EMs differing behavior in matrix and embedded contexts, etc.). They explicitly mention data not unlike our own as a reason to posit such an analysis, expressing an intuition that both $B$ and $B^{\prime}$ are felicitous answers to A. A: That might have been a mistake. B: Yes, it might have been. 
$B^{\prime}: Y e s$, it was. We propose that this is true of all subjective judgments, which then means that this claim extends to PPTRs. This might seem strange at first given that PPTRs are thought to have a more restrictive range of meaning than PPTs but that PPTs, as general judgments, only push one proposition to the table (after all, they are supposedly explicitly relativized). However, much as the $p$ in might $p$ is considered a prejacent, the fact that something tastes good is a proper subpart of a PPTR sentence where something tastes good to the speaker. Because a cancellation of $p$-gen entails $\neg p$-gen and vice versa, there will always be at least one proposition in addition to $p$-ind that is put in the DC, pushed onto the Table and can be a target for negation. We argue that this the real difference between English and Spanish: English (often?) implicates $\neg$ p-gen by default with PPTRs, whereas Spanish (often?) implicates $p$-gen. We usually think of implicature calculation as being universal, but there are a number of ways to compute the relevance of one sentence to another, and these can be opposed. When someone says that something tastes good to her, one could think that the extra effort involved in relativizing to herself signals that she does not think it holds more generally, or one could think that people often assume their own preferences are shared by others and that her statement is being used to establish good taste. If, for example, one of these calculations is made more often than the other by some group of speakers, it could become a generalized conversational implicature (GCI), and it is then plausible to think that two different GCIs could form in different populations.

One piece of evidence potentially supporting this idea is the following: English speakers, as per our results, cannot refute $p$-gen after the utterance of a PPTR. However, though it was not tested in the original experiment, it seems that $\neg p$ gen is refutable in English in the same environments were $p$-gen didn't work, as in A: The soup tastes good to me. B: No, other people like it, too. The natural question is then whether Spanish speakers, as a whole or in certain dialects, find dialogues like this one infelicitous. One of our reviewers, a native speaker of a third Spanish dialect, does find it infelicitous, though the second author of this paper does not. A few Uruguayan Spanish speakers were consulted and found the dialogue odd, but we hope to do further follow-up work on this point. In the meantime, it seems that the linguistic difference among English and Spanish with respect to PPTRs could boil down to a difference in the default polarity of an implicature.

In review, the following are our proposed update rules for each meaning type we tested in our experiment:

- BA: $p$ is added to the speaker's DC and pushed to the Table.

- PPT: $p$-gen is added to the speaker's DC, as is $p$-ind (unless cancelled). Only $p$-gen is pushed to the Table.

- EM: $\diamond p$ is added to the speaker's DC. $p$ and $\diamond p$ are pushed to the Table.

- PPTR: $p$-ind is added to the speaker's DC as well as either $p$-gen or $\neg p$-gen. Both $p$-ind and $p$-gen/ $\neg p$-gen (whichever is in the DC) are pushed to the Table. 
- EMR: neither $p$ nor $\neg p$ are added to the speaker's DC, but the proposition that the speaker has no knowledge of $p(q)$ enters the DC. Neither $p$ nor $\neg p$ exists in the DC to be pushed onto the Table, but $q$ does and is.

With this theory, we do not need to postulate that $N o$ works differently in the two languages, nor that $N o$ targets different types of content across languages. And under this analysis, it is just a fluke that PPTRs and EMRs are both unable to be directly refuted in English because there are different reasons posited for their non-refutability. The non-refutability of EMRs is absolute in the sense that they never introduce $p$ or $\neg p$. We predict that this is true cross-linguistically, matching our results. With PPTRs, on the other hand, the lack of refutability has to do with the fact that their $p$-gen or $\neg p$-gen meaning blocks the possibility of denying the other. This is something we expect to vary cross-linguistically, and, as such, we argue against a parallel between PPT and EM.

\section{Conclusion}

In this paper, we have presented the results of an experimental study collecting naturalness judgments from English and Spanish speakers to determine whether PPTs and EMs can be refuted in dialogue. We validated the hypotheses of the philosophical/linguistic literature on faultless disagreement in English, but we also showed that PPTs and EMs do not pattern alike with respect to refutation cross-linguistically when relativized. We argued against the possibility that these effects were primarily due to differences in politeness norms across cultures by replicating our results for another dialect of Spanish, and we argued for an explanation in terms of pragmatic differences. We showed how such differences could arise on the basis of a simple polarity difference in the implicature drawn from a relativized PPT, and we specified the discourse function of each type of meaning separately, also drawing out the patterns that they share. Further work is naturally required to decide among the various theoretical consequences of these results, which we are in the process of conducting. We are particularly interested in looking for similarities and differences within the class of evaluative items, separating aesthetic and taste predicates as [21] has done. This article represents one of the first steps in the domain of psycholinguistic philosophy/pragmatics on this subject, and we believe that it shows the benefits of an experimental approach in, for example, teasing apart EMRs and PPTRs very clearly, showing that they should not be given a unified analysis.

Acknowledgments. This research has been funded in part by the Conseil de recherches en sciences humaines du Canada (430-2013-000804), EURO-XPRAG, the Juan de la Cierva Program (JDC-2009-3922), the Ramn y Cajal Program (RYC-2010-06070), and by project FFI2012-34170 (MINECO). We also thank our three anonymous reviewers whose comments were extremely useful. 


\section{References}

1. Umbach, C. Evaluative propositions and subjective judgments. In J. van Wijnbergen-Huitink C. Meier (eds.), Subjective meaning. Berlin, de Guyter. (In press, to appear in 2015)

2. Farkas, D., Bruce, K. On reacting to assertions and polar questions. Journal of Semantics 27.1. (2010)

3. Kölbel, M. Faultless disagreement. Proceedings of the Aristotelian Society (Hardback), Vol. 104.1. Blackwell. (2004)

4. Stojanovic, I. Talking about taste: disagreement, implicit arguments, and relative truth. Linguistics and Philosophy 30.6. (2007)

5. Stephenson, T. Judge dependence, epistemic modals, and predicates of personal taste. Linguistics and Philosophy 30.4. (2007)

6. Lasersohn, P. Context Dependence, Disagreement, and Predicates of Personal Taste. Linguistics and Philosophy 28.6. (2005)

7. Pearson, H. A judge-free semantics for predicates of personal taste. Journal of Semantics 30.1. (2013)

8. Egan, A. Epistemic modals, relativism and assertion. Philosophical Studies 133.1. (2007)

9. von Fintel, K. Would you believe it? The King of France is back! Ms. (2004)

10. von Fintel, K., Gillies, A. An opinionated guide to epistemic modality. Oxford studies in epistemology 2. (2007)

11. Roberts, C. Information structure in discourse: Towards an integrated formal theory of pragmatics. Semantics and Pragmatics 5.6. (2012)

12. Simons, M., Tonhauser, J., Beaver, D., Roberts, C. What projects and why. SALT Proceedings. (2011)

13. Castroviejo, E., Mayol, L., and Smith, E. Conventional implicatures and direct refutation. Ms. (2014)

14. Grice, H. P., Cole, P. Morgan, J. Syntax and semantics. Logic and Conversation 3. (1975)

15. Team, R. Core. R: A language and environment for statistical computing. R Foundation for Statistical Computing, Vienna, Austria, 2012. (2014).

16. Bates, D, Maechler, M., Bolker, B. lme4: Linear mixed-effects models using S4 classes. R package version 0.999999-0. 2012. (2013)

17. Gunlogson, C., and G. Carlson. Predicates of experience. Subjective Meaning. In J. van Wijnbergen-Huitink C. Meier (eds.), Subjective meaning. Berlin, de Guyter. (In press, to appear in 2015)

18. Reiter, R. A contrastive study of conventional indirectness in Spanish: Evidence from Iberian and Uruguayan Spanish. Pragmatics 12.2. (2002)

19. Barker, C. The dynamics of vagueness. Linguistics and Philosophy 25.1. (2002)

20. Bylinina, L. The Grammar of Standards: Judge-dependence, Purpose-relativity and Comparison Classes in Degree Constructions. PhD dissertation. LOT Dissertation Series 347. (2014)

21. McNally, L., and Stojanovic, I. Aesthetic Adjectives. In J. Young (ed.), Semantics of Aesthetic Judgment. Oxford University Press. (To appear). 